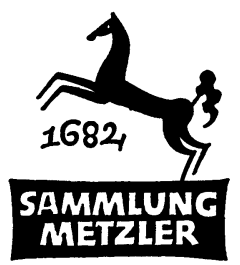

REALIENBUCHER FUR GERMANISTEN

ABT. D:

LITERATURGESCHICHTE 


\section{GOTTFRIED BENN}

2., überarbeitete und ergänzte Auflage

MCMLXX

J. B. METZLERSCHE VERLAGSBUCHHANDLUNG STUTTGART 
r. Aufl. (r.-5. Tsd) 1962

2. Aufl. (6.-10. Tsd) 1970

M 26

ISBN 978-3-476-10026-9

ISBN 978-3-476-03809-8 (eBook)

DOI $10.1007 / 978-3-476-03809-8$

(C) Springer-Verlag GmbH Deutschland 1962

Ursprünglich erschienen bei J. B. Metzlersche Verlagsbuchhandlung und Carl Ernst Poeschel Verlag GmbH in Stuttgart 1962 


\section{VOR WORT}

Dieser Band gibt, soweit der durch die "Sammlung Metzler" gesteckte Rahmen und die Forschungslage es erlauben, eine umfassende und möglichst genaue Orientierung über Leben und Werk Gottfried Benns. Die Vermittlung von Daten und Fakten steht dabei im Vordergrund. Es mußten aber auch, vor allem in der Darstellung der Lyrik, die einzelnen Stufen und Linien der Entwicklung Benns als Dichter erst herausgearbeitet werden, sowohl formal wie thematisch.

Mein ganz besonderer herzlicher Dank gilt Frau Dr. Ilse Benn, die mit unermüdlicher Geduld alle biographischen Daten mit mir nachprüfte und mir manche bisher unbekannte Einzelheiten aus Benns Leben zugänglich machte, sowie dem Verleger Benns, Herrn Max Niedermayer $\dagger$, der meine Arbeit sehr bereitwillig gefördert hat.

Die Literaturangaben der Lohnerschen Bibliographie, auf die bei jedem Werk verwiesen wird, habe ich weitgehend nachzuprüfen versucht, vor allem in Zusammenarbeit mit Herrn Fritz Werner (Freiburg i. Br.), dem ich auch an dieser Stelle für die Großzügigkeit danken möchte, mit der er mir die reichen Sammlungen seines Benn-Archivs geöffnet und mich auf bisher noch nicht bekannte Drucke und Veröffentlichungen aufmerksam gemacht hat. Wesentliche Korrekturen verdanke ich auch Herrn Professor Dr. Reinhold Grimm, Herrn Professor Dr. Edgar Lohner, Herrn Dr. Paul Raabe und Herrn Dr. Joachim W. Storck. Die Literaturangaben sind bis 1970 weitergeführt.

Universität Kiel, im Juli 1970

FriedRICH WILHELM WODTKE 


\section{INHALT}

VORBEMERKUNGEN UND LITERATURÜBERSICHT . . . . . I I

LEBEN UND WERKE BENNS

I. Jugend und erste Berliner Zeit (1886-1914) . . . . Io

II. Die Brüsseler Zeit (I9I4-1917) . . . . . . . . . 2 I

III. Berlin (1917-1932) . . . . . . . . . . . . . . 27

IV. Berlin (1933-1934) . . . . . . . . . . . . . . 47

V. Hannover, Berlin und Landsberg an der Warthe (1935-1945) . . . . . . . . . . . . 63

VI. Spätzeit in Berlin (1945-1956) . . . . . . . . 77

Aufgaben und Probleme der Benn-Forschung . . . 102 REgISTER . . . . . . . . . . . . . . . . . . . II 3 


\section{AbKüRZUngen}

DVjs. = Deutsche Vierteljahresschrift für Literaturwissenschaft

EG und Geistesgeschichte

$=$ Etudes Germaniques

$\mathrm{FAZ}=$ Frankfurter Allgemeine Zeitung

GR = The Germanic Review

GRM = Germanisch-Romanische Monatsschrift

GW = Gesammelte Werke:s.S. I

NZZ = Neue Zürcher Zeitung

PMLA = Publications of the Modern Language Society (USA)

RGG = Religion in Geschichte und Gegenwart

Wirk Wort $=$ Wirkendes Wort

ZfdPh. $\quad=$ Zeitschrift für deutsche Philologie

Die nur mit den Verfassernamen zitierten Werke findet man auf S. 2-9. 


\section{VORBEMERKUNGEN \\ UND LITERATURÜBERSICHT}

I. Manuskripte, Typoskripte und Erstdrucke werden im Anmerkungs- und Lesarten-Apparat in den 4 Bden der "Gesammelten Werke« $(=\mathrm{GW})$ sowie in der Benn-Bibliographie von Edgar Lohner (= Lohner I) mitgeteilt, in beiden Fällen nicht immer zuverlässig (s. u.). Doch muß hier aus Raumgründen auf beide verwiesen werden. Die nachgelassenen Manuskripte, Skizzenbücher, Briefwechsel und Tagebücher befinden sich im Besitz von Frau Dr. Ilse Benn, StuttgartBernhausen, die alle Urheberrechte besitzt und im Deutschen Literaturarchiv in Marbach ein "Benn-Archiv " eingerichtet hat; wichtige, z. T. noch unveröffentlichte Benn-Briefe besitzen Dr. F. W. Oelze in Bremen, Paul und Gertrud Hindemith u. a.; außerdem tauchen immer wieder Briefkonvolute auf. Für die Benn-Forschung außerordentlich wichtig ist die im wesentlichen unvensehrt erhaltene Bibliothek des Dichters mit seinen Erstausgaben und Handbüchern, seinen Schulbüchern und Erwerbungen der Jugend- und Studienzeit sowie Dedikationsexemplaren vieler Expressionisten bis hin zu Bert Brecht; es fehlen lediglich einige von Benn in Landsberg an der Warthe benutzte Bücher, wie Erwin Rohdes »Psyche«, Dostojewskis »Schuld und Sühne «, der 2. Band der Trilogie "Die Götvinnen « von Heinrich Mann usw. Das umfangreichste Benn-Archiv besitzt FrIȚZ Werner in Freiburg im Breisgau..

II. Auf die Erstdrucke und Einzelausgaben, Vorabdrucke und erste Sammlungen wird bei den jeweiligen Werkbehandlungen am Schluß der betreffenden Kapitel hingewiesen. Für die wissenschaftliche Beschäftigung mit Benns literarischer Tätigkeit in allen ihren Äußerungen ist vorläufig maßgebend:

GotTfried Benn: Ges. Werke, hrsg. v. Dieter Wellershoff. 4 Bde.

Wiesbaden: Limes Verlag. Bd I (= GW I): Essays, Reden, Vorträge. 1959; Bd 2 (= GW II): Prosa und Szenen. 1958; Bd 3 (= GW III): Gedichte. 1960; Bd 4 (= GW IV): Autobiographische und vermischte Schriften. I 961 .

-: Ges. Werke in 8 Bden, hrsg. v. Dieter Wellershoff. Paperback-Ausgabe. Ebda 1967, 21968. (Text identisch mit der vierbändigen Ausgabe, mit einigen Ergänzungen). (Rez: Opitz K., Germanistik ı०, 1969, S. 172.)

Die verdienstvolle Ausgabe bringt alle veröffentlichten und viele unveröffentlichte Texte, die innerhalb der vom Herausgeber bestimmten, nicht immer überzeugenden Sachgruppen chronologisch geordnet in der Fassung letzter Hand erscheinen. Die Streichungen und Textvarianten werden im Anhang, leider nicht immer vollständig und 
zuverlässig, abgedruckt. Am wenigsten befriedigt Bd 3 , da er die »Ges. Gedichte* (1956) mit ihrer die Chronologie zerstörenden Anordnung Benns unverändert abdruckt und erst im Anhang den umfangreichen Rest veröffentlichter oder aus dem Nachlaß stammender Gedichte chronologisch ordnet. Dadurch werden viele Gedichtzyklen auseinandergerissen, und die Ubersicht über die Entwicklung der Lyrik Benns wird unnötig erschwert. Die Anmerkungen verzichten auf viele Einzelheiten früherer Varianten und sind nicht immer zuverlässig. Das Oratorium »Das Unaufhörliche* wird im ersten Teil unvollständig, im Anhang noch einmal vollständig abgedruckt; die in "Primäre Tage* bereits veröffentlichten Gedichtentwürfe, Aphorismen und Notizen Benns sowie die wertvollen Anmerkungen der Herausgeberin sind nicht aufgenommen worden. Dieser Band bedarf dringend einer Umarbeitung, desgleichen das Register der Gedichtüberschriften. Wertvoll ist das Begriffsregister am Ende von Bd 4; ein Namensregister ist notwendig und für die nächste Auflage dringend erwünscht. (Rez.: GosE, W., Germanistik 2, 196I, S. 276 f.; HilsBECHER, W.: Das große lyrische Ich. In: Frankf. Hefte Is, 1960, S. 437-439; Schonauer, F., Der Monolog eines Intellektualisten (G. B.), Dt. Rundschau 86, 1960, S. 890-894; BlöCKER, G., Süddt. Ztg $\mathrm{Nr}_{144}$, I6/17. Juni 1962, FAZ $\mathrm{Nr}$ 191, 18. Aug. 1962; ENZensberger, H. M., Der Spiegel 16, Nr 23, 6. Juni 1962, S. 74).

GotTfried BenN: Medizinische Schriften. Hrsg. u. mit e. Nachwort v. Werner Rübe. Wiesbaden: Limes Verlag 196s.

Gotrfried Benn: Den Traum alleine tragen. Neue Texte, Briefe, Dokumente. Hrsg. v. Paul Raabe u. Max Niedermayer. Wiesbaden: Limes Verlag 1966. Neuausgabe: dtv-Taschenbuch $\mathrm{Nr}$ 557, 1969. (Rez.: ExNer, R: Jenseits von Sieg und Niederlage. G. B. - zehn Jahre nach seinem Tod. In: Die Zeit Nr 31, 29. Juli 1966, S. I3; Roggeman, W.: Een Benn-boek tien jaar na zijn dood; Kritisch analytische opmerkingen bij $»$ Den Traum alleine tragen «. In: Dietsche Warande en Belfort II I, 1966, S. 694-704; Grimm, R.: Germanistik 8, 1967, S. 4 I0.)

Das Gottfried Benn-Buch. Eine innere Biographie in Selbstzeugnissen. Hrsg. v. Max Niedermayer u. Marguerite Schlüter. (Fischer Bücherei $\mathrm{Nr}$ 923), 1968.

Dichter über ihre Dichtungen. GotTrRied BenN. Hrsg. v. Edgar Lohner. München: Heimeran 1969.

GotTfried Benn: Texte aus dem Nachlaß (1933-1955). Hrsg. v. Harald Steinhagen. In: Schiller-Jb. I 3, 1969, S. 98-1 14.

\section{Auswablen:}

Gottrried Benn: Lyrik und Prosa, Briefe und Dokumente. Eine Auswahl, hrsg. v. Max Niedermayer. Wiesbaden: Limes Verlag 1962. (= Limes -PAPERBACK.)

GotTfried Benn: Auswahl aus dem Werk, hrsg. v. Dieter Wellershoff. Darmstadt: Moderner Buch-Club 1962.

GotTfried Benn: Seleoted Poems. Edit. with introduction, inter- 
pretation and notes by Friedrich Wilhelm Wodtke. Oxford Press, Clarendon Series, 1970.

Ungesicherte Texte: Im Benn-Archiv'von Fritz Werner, Freiburg: »Dichtung auf Bestellung «, "Brieffragment an ein Mitglied der Preuß. Akademie der Künste .

\section{Brief-Ausgaben und -veröffentlichungen:}

Ausgewählte Briefe (= ABr.). Mit Nachw. v. Max Rychner. Wiesbaden: Limes 1957; ${ }^{2}$ 1959. - Literatur: RYCHNER, M.: G. B. in seinen Briefen. In: Neue Dr. Hefte 4, 1957/58, S. 487-497. Ders.: Die Briefe G. B.s. In: M. R. Antworten, 196I, S. 293-312. Ders.: Die Briefe G. B. s. In: M. R., Aufsätze zur Literatur, 1966, S. 471-495. Hesse, H.: Uber G. B.s. Briefe. In: Poesie u. Prosa, Limes No 4, Sept. 1960, neu in: Limes-Paperback, 1962, S. LXXXIX. НоноғF, C.: B.-Briefe. In: Merkur I2, 1958, S. 689-691. Ihlenfeld, K.: Gewagtes Leben. B.s. Briefe. In: Zeitgesicht, 196I, S. 35I-354. BlöCKER, G.: Krit. Lesebuch, 1962, S. I21-125. FRITZ, W. H.: B. in seinen Briefen. In: Streit-Zeit-Schrift H. V, I, Juli 1964, S. 33 bis 34 .

Briefe an Ernst Jünger, E. R. Curtius, Max Rychner u. a. Zürich: Verlag Die Arche 1960. (Die kleinen Bücher der Arche. Nr. 3 1 1/1 2.)

Briefwechsel mit Max Rychner. In: Merkur I I, 1957, S. 850-857.

Briefe an Carl Werckshagen. In: Limes-Lesebuch, 2. Folge, 1958, S. 43 bis 61 ; vgl. Limes-Paperback S. 72, 75 .

Briefe an seine TochterNele. In: Nele P. Soerensen, Mein Vater G. B. Wiesbaden: Limes Verlag 1960, passim.

Briefe an Dr. Julius Gescher u. Dr. Jürgen Eggebrecht. In: Dr. Pfarrerblatt 6I, 196I, S. 2 18-220. Neu in: G. B., Den Traum alleine tragen, 1966, S. 264-21 3 .

Briefe an Adolf Frisé. In: Notizen (Studentenztg. f. Stuttgart u. Tübingen), 5, I961.

Briefe an Dr. F. W. Oelze. In: Merkur I5, 196I, S. 438-454. Mit Vorwort von Dr. Oelze. Ferner in: Limes-Paperback, 1962, S. 122 bis 124, I3 I-132, I59-161, I69 (s. u. S. 4.).

Brief an Max Krell (12. Juni 1920). In: Max Krell, Das alles gab es einmal, 1961, Facsimile nach S. I 12.

Brief an Fritz Sorge (17. Jan. 1932). In: Limes Paperback S. I08. Briefe an Ellinor Büller-Klinkowström (1935-1937). In: ebda S. 125 bis 126, 134-36. (Vgl. K. K., FAZ Nr. 169, 24. Juli 1962.) Brief an Paul Fechter (23. März 1944). In: ebda S. is8. Brief an Jobannes Weyl (5. Okt. 1946). In: ebda S. 163-168. Briefe an Max Niedermayer (1948-195I). In: ebda S. I7I, 225-228, 24I-245. Brief an Gert Micha Simon (I I. Sept. 1949). In: ebda S. 235. Briefe von Ernst Robert Curtius an G. B. (1948-1950). In ebenda S. XVI-XX. Brief von George Grosz an G. B. (17. Nov. 1949). In: ebda S. XXI.

Brief an Walter Lennig (14. Juni 1956). In: Lennig: G. B., 1962, S. I 52. Brief an Georg Britting (4. Mai 1954). In: D. Bode, G. Britting, 1962, S. II4. 
Gottraried Benn: Das gezeichnete Ich. Briefe aus den Jahren r 900 bis 1956. (dtv Nr 89), 1962 (neu aufgenommen Briefe an Dr. F. W. Oelze, Adolf Frisé, Carl Werckshagen).

Briefe an Wolfgang Bächler (20. Nov. 1949, 29. Mai 1950): In: R. Grimm, Strukturen, 1963, S. $351-352$.

Brief an Hans Bender (17. Aug. 1954). In: Akzente 10, 1963, S. 212 Facsimile.

Briefe an Kasimir Edschmid und Karl Otten. In: K. Edschmid, Briefe der Expressionisten, 1964, S. 88-90, 162.

Brief an Adolf Richard Meyer (27. Juli 1914). In: Jürgen P. Wallmann, Ein Benn-Fund. In: Neue Deutsche Hefte $10,1964, \mathrm{Nr} 98$, S. I 82-1 84. Neu in: G. B. Den Traum alleine tragen, 1966, S. 2 I-22. Brief an Börries von Münchbausen (Is. 10. 1933). In: Paul Raabe (Hrsg.): Expressionismus. Der Kampf um eine literarische Bewegung, I 965 .

Briefe an Carl Schmitt (28. März I 943, I. Sept. 1943): Piet Tommissen: A propos de deux lettres inédites de Gottfried Benn. In: EG 20, 1965, S. $573-576$. Neu in: G. B., Den Traum alleine tragen, 1966, S. $214-217$.

Briefe an Max Niedermayer. In: Briefe an einen Verleger. Max Niedermayer zum 60. Geb. Hrsg. v. Marguerite Schlüter, 1965.

Briefe an Albert Ebrenstein und Kurt Wolff (1916-17). In: Kurt Wolff, Briefwechsel eines Verlegers, 1966. Neu in: G. B., Den Traum alleine tragen, 1966, S. 24-29.

Briefe an Herwarth Walden, Alfred Richard Meyer, Albert Ebrenstein, Kurt Wolff, Max Krell, Else Lasker-Schüler, Tilly Wedekind, Pamela Regnier-Wedekind, Kaethe von Porada, Wilhelm Lehmann, Ellinor Büller-Klinckowström, Julius Gescher, Jürgen Eggebrecht, Leonharda Gescher, Carl Schmitt, Alain Bosquet, Marguerite Schlüter, Akademie der Künste Berlin. In: G. B., Den Traum alleine tragen, 1966.

Brief von Kurt Hiller an G. B. (16. Sept. 1951). In: Poesie und Prosa, Mitteilung des Limes Verlag, März 1968.

Briefe an Dr. F. W. Oelze, Egmont Seyerle u. a. In: Dichter über ihre Dichtungen. G. B. Hrsg. v. E. Lohner, 1969.

Briefe an Dr. F. W. Oelze. In: Harald Steinhagen, Die Statischen Gedichte G. B.s, 1969 .

\section{Gespräche und Interviews:}

Das Volk und der Dichter. Rundfrage der Dt. Allg. Ztg. In: Die Literatur 35, 1932/33, S. 676.

Grigol Robakidse, G. B. u. Adolf Frisé: Mythos, einst und jetzt. Ein Dreigespräch. In: Dt. Zukunft (Berlin), 3. Juni 1934; Teilabdruck in: Die Literatur 36, 1933/34, S. 641.

KeIL, Helly: Menschen unserer Zeit: Der Dichter. Ein Interview mit G. B. In: Germania (Berlin), 23. Sept. 1934.

Lind, Georg Rud.: Interview mit G. B. In: Die Tat (Zürich), I4. Jan. 1950, S. I 3. Lit.: H.-D. BAlser, S. 209. 
Michael, Peer: Der Dichter aus der Doppelwelt. Gespräch mit G. B. In: Der Fortschritt (Düsseldorf), 22. Mai I953.

Christlieb, W.: Es liegt etwas vor ... Gespräch mit G. B. In: Abendztg (München), 9. März 1954.

\section{Die Stimme Benns ist auf Langspielplatten festgehalten:}

I. G. B. liest $\gg$ Totenrede auf Klabund " (histor. Aufnahme). Wiesbaden: Limes $1958.17 \mathrm{~cm}$.

2. G. B. liest "Die neue literarische Saison* (histor. Aufnahme) und Gedichte. Wiesbaden: Limes i $960.25 \mathrm{~cm}, 33 \mathrm{UpM}$.

3. G. B. liest Urgesicht u. Gedichte. Wiesbaden: Limes. 1961. Nr $007421.25 \mathrm{~cm}, 33 \mathrm{UpM}$.

4. G. B. liest Gedichte und Prosa. Dt. Grammophon-Ges. Nr $4 \mathrm{I} 003$ LPS. $25 \mathrm{~cm} .33$ UpM.

5.G. B. liest *Altern als Problem für Künstler«. Wiesbaden: Limes $1957.30 \mathrm{~cm}, 33 \mathrm{UpM}$.

6. vgl. ferner: *jazz und lyrik", Gedichte v. G. B. / Musik v. J. J. Johnson u. Kai Winding/Sprecher Gert Westphal. Hamburg: Philips B 47059 L. $30 \mathrm{~cm}, 33$ UpM.

\section{Bibliographien:}

(ohne Verf.): Kleine Bibliographie G. B. In: Das Antiquariat (Wien) I2, I 956, S. I 190-I9I.

Lohner, Edgar: G. B. Bibliographie / Bibliographie 1912-1955, Phibiblion I, 1957, H. I, S. 59-79; dasselbe vollständiger und mit Sekundärliteratur als:

LOHNER, EDGAR: G. B. Bibliographie 1912-1956. Wiesbaden: LimesVerlag 1956; 2., verm. Aufl. I960.

Die für wissenschaftliche Arbeiten über B. unentbehrliche Bibliographie verzeichnet I. die Lyrik, 2. die Prosaveröffentlichungen G.B.s, 3. die Úbersetzungen der Werke G. B.s, 4. Aufsätze, Besprechungen und Hinweise in Büchern, Literaturgeschichten, Zeitschriften, Zeitungen und Lexika zum Werk G. B.s (wobei manches Unwesentliche fehlen könnte und vieles zu ergänzen wäre), s. Fremdsprachige Aufsätze, Besprechungen und Angaben zum Werk G. B.s (die mit 4. vereinigt werden sollten, zumal of die gleichen Verfasser unter 4. und 5. erscheinen) und schließt mit einem Verfasser- und Ubersetzerverzeichnis sowie einem Sach- und Namensverzeichnis. Bedauerlich ist die weitgehende Unzuverlässigkeit der Angaben in sämtlichen Abschnitten; eine verbesserte und vermehrte Neuauflage ist ein dringendes Desiderat. - Nach dieser Bibliographie wird im Folgenden mit Angabe der entsprechenden Nrn zitiert. Soweit möglich, wurden fehlerhafte Angaben korrigiert und im Benn-Archiv von Fritz Werner nochmals nachgeprüft.

Köttel wesch, Clemens: Bibliographie der dt. Literaturwiss. Bd II bis VIII, 1954-1968, Frankfurt: V. Klostermann 1958-1969, passim. 
Paetel, Karl O.: G. B. im Lichte der amerikan. Kritik. In: Neue Dt. Hefte 6, 1954, S. 475-478. Ders.: Auslandsstimmen über G. B. In: Edkart 28, 1959, S. 83-86. (Rez.: B. Hillebrand, Literaturwiss. Jb. NF 5,1964, S. 406 f.)

Ноноғ, Curt: Die Erforschung G. B.s In: Merkur I 3, 1959. S. 893/5. Buddeberg, Else: Probleme um Gottfried Benn I/II. In: DVjs. 34, 1960, S. 107-161; 35, 1961, S. 433-479; beides zusammen auch als Sonderausgabe (Referate aus d. DVjs.) 1962. = BUDDEBERG II.

SCHUBERT, Werner: (Drei westdeutsche Benn-Monographien [Nef, Klemm, Wellershoff]). In: Weimarer Beiträge 7, 196 I, S. $645-65$ I.

GRIMm, ReINHOLD: Kritische Ergänzungen zur Bennliteratur. In: R.G., Strukturen, 1963, S. 273-352.

Hillebrand, Bruno: G. B. im Spiegel der Literatur. In: literaturwiss. Jb. N.F. S, I 964, S. $381-426$.

Märkel, Friedrich: Die Uberwindung des Nihilismus durch die Kunst. Zur neueren Literatur über G. B. In: Zeitwende 36, 1965, S. $528-534$.

WallmanN, Jürgen: Kunst als metaphysische Tätigkeit: Hinweise auf die jüngste Benn-Literatur. In: Die Tat (Zürich) 2. Juli 1966, S. 33 bis 34. Neu in: J. W.: Argumente, 1968, S. I87-195.

WaLlmanN; JürgEN: Betrachtet, forscht, die Einzelheiten sammelt ... In: Die Welt der Literatur $\mathrm{Nr}$ is, 17. Juli 1969.

Ders.: Neue Literatur zum Werk G. B.s. In: Die Tat (Zürich) 2. Aug. 1969.

VIII. Monographien (nach ihnen wird im folgenden nur mit Verfassernamen und Seitenzahlen zitiert):

Hamecher, Peter: Entformung und Gestalt. G. B. - Stefan George. 1932. S. 7-20. (Rez.: F. Maraun, Kritische Gänge der Berl. Börsen$\mathrm{Ztg}$, 28. Febr. 1932).

Косн, Tнilo: G. B. Ein biogr. Essay. 1957. (Rez.: C. Hohoff, Merkur I3, I 959, S. 894; E. Buddeberg II, S. 7-8; F. Wood, GR 35, 1960, S. 301-304; H. Meixner, Archiv f. d. Stud. d. neueren Sprachen 200,1963 , S. 214-2 I 5; B. Hillebrand, Literaturwiss. Jb. N.F. s, 1964, S. 389 ).

Schümann, Kurt: G. B. Eine Studie. 1957. (Rez.: P. Garnier, EG I3, 1958, S. 384-385; C. Hohoff, Merkur 1 3, 1959, S. 894; Buddeberg II, S. 6-7).

Grimm, Reinhold: G. B. Die farbliche Chiffre in der Dichtung. I958. 21962. (Rez.: R. Geissler, Wirk Wort 9, 1959, S. 252; C. Hohoff, Merkur I 3, 1959, S. 894; P. Garnier, EG 14, 1959, S. 28 I; E. Buddeberg II, S. 39-40; B. Hillebrand, Literaturwiss. Jb. N.F. S, 1964, S. 405.$)$

Klemm, Günther: G. B. 1958. (Rez.: C. Hohoff, Merkur 13, 1959, 
S. 894; E. Buddeberg II, S. I0-I I P. Garnier, EG I6, I96 I, S. 188; W. Schubert, Weimarer Beiträge 7, 1961, S. 647-648; B. Hillebrand, Literaturwiss. Jb. N.F. 5, 1964, S. 403-404.)

Nef, Ernst: Das Werk G. B.s. 1958. (Rez.: C. Hohoff, Merkur I3, 1959, S. 895; E. Buddeberg II, S. 31-34; W. Schubert, Weimarer Beiträge 7, 1961, S. 645-647; B. Hillebrand, Literaturwiss. Jb. N.F. 5, 1964, S. 392-394.)

Wellershoff, Dieter: G. B. Phänotyp dieser Stunde. Eine Studie über den Problemgehalt seines Werkes. 1958, Ullsteinbuch $\mathrm{Nr} 499$, 1 964. (Rez.: C. Hohoff, Merkur 13, 1959, S. 895; E. Buddeberg II, S. 8, 44-48; F. Wood, GR 35, 1960, S. 301-304; R. Montigny, EG I6, 196I, S. 74; W. Schubert, Weimarer Beiträge 7, 196I, S. 648 bis 65 I ; B. Hillebrand, Literaturwiss. Jb. N.F. 5, 1964, S. 394-398, vgl. S. 399-404.)

Garnier, Pierre: G. B. Un demi siècle vécu par un poète allemand. Paris I959. (Rez.: R. Grimm, Strukturen, 1963, S. 287-294.)

Pensa, Mario: Un sacerdote dell'assoluto: G. B. Bologna 1960. (Rez.: R. Grimm, Germanistik 2, 1961, S. 450 u. in: R. Grimm, Strukturen, 1963 , S. 277-286.)

Soerensen, Nele P.: Mein Vater G. B. 1960. (Rez.: E. Buddeberg II, S. $57-60$.)

Buddeberg, Else: G. B. 1961. (Rez.: R. Montigny, EG 16, 1961, S. 74; E. Lohner, DVjs. 35, 196I, S. 634-642; I. Parry, Erasmus I4, 196I, S. 743-76r ; D. Paisey, German Life and Letters I s, I 96 I/62, S. 324 bis 327; R. Grimm, Strukturen, 1963, S. 325-346; G. Guder, Modern Language Review 58, 1963, S. 143-147; J. Seidler, Monatshefte 55 , 1963 , S. 282-284; B. Hillebrand, Literaturwiss. Jb. N.F. 5, I 964, S. 407-4 I 2; N. Hinske, Philosoph. Rundschau I I, I 964, S. 26 I bis 278; J. W. Storck, Archiv f. d. Studium d. neueren Sprachen 201, 1964, S. 60-62.) = E. BUDDEBERG I.

Lohner, EdGar: Passion und Intellekt. Die Lyrik G. B.s. I96r. (Rez.: R. Grimm, Strukturen, 1963, S. 305-324; G. Guder, Modern Language Review 58, 1963, S. 143-144; H. G. Hannum, German Quarterly 36,1963 , S. $302-303$; B. Hillebrand, Literaturwiss. Jb. N.F. 5, I964, S. 4 I $5-42$ I.)

Loose, Gerhard: Die Ästhetik G. B.s. 1961. (Rez.: Th. O. Brandt, German Quarterly 35, 1962, S. 534-535; H. Plard, EG 17, 1962, S. 494-495; R. Exner, Monatshefte 55, 1963, S. 42-44; R. Grimm, Strukturen, 1963, S. 295-305; H. G. Hannum, Modern Language Quarterly 24, 1963, S. 1 14-116; H. Wocke, Journal of English and Germanic Philology 62, 1963, S. 196-197; B. Hillebrand, Literaturwiss. Jb. N.F. 5, I 964, S. 41 3-4 Is; N. Hinske, Philosoph. Rundschau II, 1964, S. 278-28I.)

Uhlig, Helmut: G. B. 1961. = H. Uhlig II. (Rez.: R. Grimm, Strukturen, 1963, S. 274-275; B. Hillebrand, Literaturwiss. Jb. N.F. s, 1964, S. 41 2-413.)

LENNIG Walter: G. B. 1962, ${ }^{2} 1963$ (rowohlts monographien. 71.) 
(Rez.: R. Grimm, Strukturen, 1963, S. 275-277; B. Hillebrand, Literaturwiss. Jb. N.F. 5, 1964, S. 389.)

Allemann, Beda: G. B. Das Problem der Geschichte. 1963 (Rez.: B. Hillebrand, Literaturwiss. Jb. N.F. S, 1964, S. $425-426$.)

Kaiser, Helmut: Mythos, Rausch und Reaktion. Der Weg G. B.s und Ernst Jüngers. 1962. (Rez.: J. P. Wallmann, Der Monat is, I963, H. I78, S. 85-86; Henri Plard, EG I 9, 1964, S. 70-73; D. Sommer, Weimaner Beiträge 10, 1964, S. 895-899.)

WODTTKe, Friedrich WILHELM: G. B. 1962, ${ }^{2} 1970=$ WodTTKE I. (Rez.: R. Grimm, Strukturen, 1963, S. 346-350; B. Hillebrand, Literaturwiss. Jb. N.F. 5, 1964, S. 422; J. Seidler, Monatshefte 56, 1964, S. 359-360; G. Bauer, Euphorion 58, 1964, S. 214 ; J. C. Middleton, German Life \& Letters I9, 1965, No I, S. 84; M. Fancelli, Rivista di Letterature Moderne e Comparate 19, 1966, N I. Th. Ziolkowski, The Germanic Review 39, 1964 , No 4, S. 66-67.

WodtKe, Friedrich Wilhelm: Die Antike im Werk G. B.s, 1963 $=$ Wодтке II. (Rez.: B. Hillebrand, Literaturwiss. Jb. N.F. S, 1964, S. 422-424; J. P. Wallmann, Der Monat I6, 1964, H. I89, S. 79-8I.)

WaLlMANN, JÜrgen: G. B., I 965 .

Masini, Ferruccio: G. B. e il mito del nichilismo, Padova 1968.

\section{Dissertationen:}

Ivask, Ivar: G. B. als Lyriker. MA Thesis University of Wisconsin, 1949.

Wellershoff, Dieter: Untersuchungen über Weltanschauung und Sprachstil G. B.s. Diss. Bonn 1952 (Masch.).

Claes, Astrid: Der lyrische Sprachstil G. B.s. Diss. Köln 1953 (Masch. 1956). (Rez.: E. Buddeberg II, S. 4I-43.)

Kaiser, Helmut: Die ideologische Entwicklung G. B.s und Ernst Jüngers. Eine Untersuchung zur weltanschaulichen Dekadenz der deutschen Bourgeoisie. Diss. Berlin (Ost) 1958; Auszug in: Wiss. Ztschr. d. Humboldt-Universität zu Berlin, Gesellschaftswiss. u. Sprachwiss. Reihe 9, 1959/60, S. s10-5II.

Haller, Elmar: Die Entwicklung der Weltanschauung G. B.s in seinem frühen Werk. Die Geschichte einer Kunsttheorie. Diss. Innsbruck 1960 (Masch.). Druck: 1965.

Hunter, Hannum G.: The Plight of Pallas: Motifs of Sterility in the Works of G. B. Diss. Harvard 1959/60.

Balser, Hans-Dieter: Das Problem des Nihilismus im Werk G. B.s. Diss. Kiel 1962 (Masch.). Druck: 1965, ${ }^{2} 1970$. (Rez.: J. P. Wallmann, Argumente, 1968, S. I 89-193.)

Liede, Helmut: Stiltendenzen expressionistischer Prosa. Untersuchungen zu Novellen von A. Döblin, C. Sternheim, K. Edschmid, G. Heym und G. B. Diss. Freiburg Br. (Masch.) 1960.

Mehl, Dietrich: Mitteilung und Monolog in der Lyrik B.s. Diss. München 196I (Masch.). 
Heimann, Bodo: Der Süden in der Dichtung G. B.s. Diss. Freiburg Br. 1962 (Masch.), Freiburg Br. (Selbstverlag) 1963.

Braun, Herbert: Wandlungen des künstlerischen Ichs bei G. B. (Untersuchungen zu einer inneren Biographie des Dichters). Diss. München 1963 (Masch.).

Steidle, Bruno: Die Erfahrung des Nihilismus und die Möglichkeit seiner Überwindung. Diss. Freiburg Br. 1963 (Masch.).

Eykmann, Christoph: Die Erfahrung des Häßlichen in der Lyrik G. Heyms, G. Trakls und G. B.s. Zur Krise der Wirklichkeitserfahrung im deutschen Expressionismus. Diss. Bonn 1964 (Masch.). Druck: 1965, ${ }^{2}$ 1969. (Rez.: J. P. Wallmann, Angumente, 1968, S. 193-195.)

Lieb, Hans Heinrich: Der Umfang des historischen Metaphernbegriffs. Diss. Köln 1964 (Masch.).

Hillebrand, Bruno: Artistik und Auftrag. Zur Kunsttheorie von B. und Nietzsche. Diss. München 1965 (Masch.), Druck: 1966. (Rez.: F. Masini, Studi germanici 4, 1966, S. 389-406); J. P. Wallmann, Argumente, 1968, S. I 87-189.)

Bleinagel, Bodo: "Absolute Prosa«. Ihre Konzeption und Realisierung bei G. B. Diss. Würzburg 1967 (Masch.). Druck: 1969.

Steinhagen, Harald: Die Statischen Gedichte von G. B. Die Vollendung seiner expressionistischen Lyrik. Diss. Tübingen 1968 (Masch.). Druck: 1969. 